\title{
Interprofessionnalité et soins intégrés: quel enseignement?
}

Olivier-Jean Glardona, Nadine Facchinetti ${ }^{b}$

a Dr méd. vet., Office fédéral de la Santé publique, Section Développement Professions de la santé; b MLaw, LL.M

Le groupe de travail «Interprofessionnalité» de la Plateforme "Avenir de la formation médicale» a publié son rapport en été 2013. Suite à de nombreuses discussions, l'importance du sujet a été reconnue et les responsables de l'enseignement développent des concepts pour l'implémentation d'un apprentissage de l'interprofessionnalité et des enseignements interprofessionnels. Cet article fait le point de la situation présente et future sur le plan national.

\section{Définitions}

Le terme Interprofessionnalité comprend deux aspects complémentaires [1], d'une part une formation interprofessionnelle (Interprofessional Education, IPE) qui mène, d'autre part, à une pratique collaborative ou interprofessionnelle (Interprofessional Collaboration, IPC) centrée sur le patient.

Selon la définition proposée par le Centre for the Advancement of Interprofessional Education du Royaume-Uni (CAIPE) [2], il y a formation interprofessionnelle lorsque deux professions ou davantage sont engagées dans un processus d'apprentissage conjoint, réciproque et qui leur permet de mieux se connaître pour mieux collaborer et améliorer la qualité des soins.

Il s'agit d'une étape nécessaire à la préparation des professionnels de la santé à une pratique collaborative, mieux à même de répondre aux besoins sanitaires locaux (collaborative practice-ready health workforces).

\footnotetext{
Bauer W. (ISFM/FMH), Brodmann-Mäder M. (Inselspital), Carruzzo P. (SWIMSA), Facchinetti N. (OFSP, présidente), Feusi E. (ZHAW), ), Frey P. (Uni BE) Glardon O.J. (OFSP, co-président), Marbet $P$. (Berner Bildungszentrum Pflege), Mesnil M. (pharmaSuisse), Schibli D. (CDS), Schwendimann R. (Basler Institut für Pflegewissenschaft), van Gessel E. (Uni GE).
}

\section{Mandat de la Plateforme}

Les expériences faites dans notre pays aussi bien dans le domaine de l'enseignement que celui de l'exercice interprofessionnel ont toutefois montré que cette vision ne suffit pas à favoriser le développement d'une collaboration interprofessionnelle efficace dans notre système de santé actuel. C'est pour aborder ce problème sur une large échelle que l'OFSP a présenté en septembre 2011 à la Plateforme "Avenir de la formation médicale» un projet de mandat, préa- lablement discuté avec la Conférence des Directeurs Sanitaires (CDS), prévoyant le lancement d'un groupe thématique «Interprofessionnalité».

La question de la priorisation de deux thèmes principaux - questions relatives à la formation ou à la répartition future des tâches et des compétences entre les professions de la santé - a été longuement débattue. La majorité de la Plateforme a décidé d'aborder le sujet sous l'angle de la formation et a formulé le mandat dans le cadre suivant:

- axer les travaux sur les questions relatives à la formation, plutôt que celles relevant de la politique sanitaire,

- développer des solutions concrètes et susceptibles d'être mises en œuvre à court terme, en priorité pour la formation médicale universitaire,

- associer ponctuellement à ses travaux des groupes d'accompagnement et un groupe de pilotage, dont la Plateforme a défini la composition.

\section{Concept du groupe thématique}

Les membres du groupe thématique* ont décidé de développer un concept d'apprentissage de l'interprofessionnalité (IPE) fondé sur des exemples concrets de collaboration interprofessionnelle (IPC), tirés des modèles de soins et de la chaîne de prise en charge des patients actuels ou prévisibles à moyen terme [3]. Ceux-ci sont principalement issus de l'exercice de la médecine de premier recours ambulatoire (tout en tenant compte des interfaces entre ambulatoire et stationnaire), centrés sur le patient. En termes d'en- 
seignement, le concept est basé sur les compétences à acquérir et orienté selon les résultats (outcomes).

Après avoir précisé les compétences dont il convenait de tenir compte en priorité en matière de collaboration interprofessionnelle (sur la base du modèle CanMEDS, commun aux professions médicales et aux métiers de la santé), et qui doivent par conséquent faire partie du catalogue des objectifs de l'IPE, les membres du groupe thématique ont développé six modèles de soins, concrétisés sous forme de modules d'apprentissages, correspondant chacun à un problème de santé et au trajet du patient dans le système de soins.

1. IPC au sein d'une structure de premier recours,

2. IPC en équipe de soins aigus, respectivement en situation d'urgence,

3. IPC «séquentielle» ou suivi ambulatoire d'un-e patient-e sorti-e d'un établissement hospitalier,

4. IPC en médecine de premier recours pour patients polymorbides, sous considération particulière des aspects "bio-psycho-sociaux",

5. IPC en réseau régional pour patients en soins palliatifs,

6. IPC à but préventif au sein d'une structure de premier recours ou un établissement médico-social.

En formulant de nombreuses propositions didactiques et pratiques pour chaque module, les membres du groupe thématique ont mis au point un instrument "clé en mains» pour un enseignement interprofessionnel, incluant des recommandations concernant les compétences et les objectifs de formation communs à toutes les professions engagées dans des pratiques collaboratives. Celui-ci propose également des recommandations méthodologiques en matière de transmission des contenus de formation (modules interprofessionnels, apprentissage en équipes) qui tiennent compte des supports existant au niveau universitaire, mais également de la systématique et des supports valables pour le niveau secondaire et tertiaire, ainsi que des différentes compétences, responsabilités et prescriptions légales en vigueur (LPMéd, LHES, LFPr, etc).

Une telle capacité collaborative ne peut être acquise sans une formation de base et une formation postgrade ciblée et coordonnée entre les professions médicales et les professions de la santé.

Ces recommandations peuvent être intégrées dans le cadre des programmes et des objectifs de formation universitaire actuels des médecins. Elles tiennent toutefois compte des mêmes critères en matière de formation de base des professions de la santé amenées à travailler avec eux de manière interprofessionnelle.

\section{Collaboration interprofessionnelle}

En dehors de la formation IPE, qui représente un prérequis à toute IPC, il est important de réunir les instruments indispensables à la réussite d'une collaboration entre partenaires professionnels de la santé dans la réalité de l'exercice des professions médicales et de la santé. On trouve de nombreuses informations sur de tels outils dans des publications internationales et nationales. Les éléments essentiels ont été groupés sous forme d'une "tool-box" pour un enseignement interprofessionnel global de la $1^{\text {re }}$ à la $6^{\mathrm{e}}$ année de médecine, permettant de développer des modules IPE progressifs (early-to-late approach) qui tienne compte du niveau de compétences croissant des étudiant-e-s et apprenant-e-s.

Cet instrument didactique devrait donner assez de liberté aux établissements de formation dans la réalisation des modules IPC pour pouvoir être adaptés selon les besoins et les possibilités de chaque faculté de médecine, et surtout être mis en place en commun avec des hautes écoles spécialisées ou des écoles professionnelles supérieures des professions de la santé de la même région.

Le présent concept représente une première étape d'introduction structurée et coordonnée de l'IPE dans les études médicales universitaires et la formation aux professions de la santé. Les experts du groupe thématique ont recommandé à la Plateforme de poursuivre l'opérationnalisation de ce concept sur le plan national dans le cadre d'un second mandat. Celui-ci devrait en outre élargir la notion de collaboration interprofessionnelle au milieu hospitalier, dans lequel elle est déjà une réalité à plus d'un titre, et aborder la question de la formation interprofessionnelle dans le cadre de la formation postgrade des professions médicales.

\section{Pratique de l'interprofessionnalité}

Ceci dit, il convient de se pencher sur l'essence même du terme d'interprofessionnalité, si on veut se donner les moyens d'en assurer la diffusion, tout en profitant de toutes les possibilités que cette pratique permet. Il s'agit en effet d'un néologisme utilisé par D'Amour et al. [4] pour décrire un concept et un cadre de référence permettant de développer «a cohesive and integrated health care practice among professionals in response to client's needs». Ce concept se dis- 
tingue de celui d'interdisciplinarité, qui concerne le développement "of integrated knowledge in response to fragmented disciplinary knowledge». Il ne correspond pas non plus au concept d'interprofessionnalisme ou de mutualisation des compétences professionnelles, avec lesquels l'interprofessionnalité est souvent confondue. Dans le premier cas, l'interaction entre les professionnels (qui implique une définition des rôles et une reconnaissance/acceptation des compétences mutuelles au sein de l'équipe en réponse au besoin d'un patient) est primordiale et elle seule permet d'obtenir le résultat fixé. Dans le second cas, chaque professionnel met ses compétences à disposition du groupe, mais l'interaction n'est pas l'élément central de la collaboration.

Selon le concept original, l'interprofessionnalité fait le lien entre les déterminants (= facteurs déterminables) et les processus de collaboration à différents niveaux, aussi en matière d'exercice que d'enseignement interprofessionnels. Par définition, l'interprofessionnalité ne peut donc correspondre à une simple juxtaposition des compétences de praticiennes et praticiens de différentes professions, fut-ce pour le bien d'un patient. C'est la nature des interactions et la manière dont elles sont structurées, qui fait la valeur du concept et permet le succès de l'exercice dans le cadre de soins intégrés.

Concrètement, l'élément essentiel de la démarche réside dans la délégation par chacun des professionnels de certaines compétences (à définir de manière interprofessionnelle et selon les besoins) à l'équipe interprofessionnelle et, en retour, dans la prise en charge d'activités professionnelles et de compétences selon des critères prédéfinis. Un des éléments centraux de cette pratique est la définition d'une vision commune et interprofessionnelle du patient et de ses besoins, et l'organisation des soins en conséquence. Pour ce faire, il est souvent nécessaire de préciser la nature du mandat attribué aux différents professionnels, à savoir le mandat de prestations (Versorgungsauftrag), mandat de prise en charge médicale (Betreuungsauftrag) et le mandat de soins (Pflegeauftrag), auquel est liée la notion de responsabilité professionnelle. Celle-ci ne devrait cependant pas être atténuée ou fragmentée par une prise en charge interprofessionnelle du patient. publique

Section Développement Professions de la sante Schwarzenburgstrasse 161 $\mathrm{CH}-3003$ Berne Tél. 0313240289 olivier-jean.glardon[at]bag. admin.ch une formation de base et une formation postgrade ciblée et coordonnée entre les professions médicales et les professions de la santé. Ce degré d'intégration des compétences et des déterminants favorisant l'interprofessionnalité n'est toutefois pas non plus réalisable sans un soutien et une coordination entre les milieux politiques, professionnels et socio-économiques. En d'autres termes, l'interprofessionnalité, pour autant qu'on veuille la développer de manière cohérente et pertinente, est l'affaire de tous les responsables et de tous les professionnels actifs dans le domaine de la santé. De même, son développement ne peut se faire de manière harmonieuse et efficace, sans vision commune du système de soins et de santé national, et par conséquent des objectifs d'enseignement qui y correspondent. Les directives du Conseil fédéral exposées dans la stratégie Santé 2020 donnent un cadre solide à une telle réflexion, en particulier en matière de soins intégrés.

\section{Et maintenant...}

C'est pour cette raison que le groupe thématique de la Plateforme a proposé dans son rapport l'organisation d'une conférence nationale, conçue de manière à servir de coup d'envoi d'une réflexion commune et interprofessionnelle dès le début. Cette manifestation a eu lieu le 4 décembre 2014. Parallèlement, l'ASSM a également organisé des rencontres d'expert-e-s et publié des documents sur la collaboration interprofessionnelle [5]. L'année 2014 peut donc être considérée comme le point de départ d'une réflexion à large échelle sur le sujet. Les membres du groupe thématique s'en réjouissent et espèrent qu'elle aura des effets tangibles et directement profitables aussi bien aux professionnels du système de santé qu'aux patients et à leurs proches.

\section{Références}

1 WHO. Framework for Action on Interprofessional Education and Collaborative Practice. 2010. http://whqlibdoc.who.int/ hq/2010/WHO_HRH_HPN_10.3_eng.pdf?ua=1

2 CAIPE: www.caipe.org.uk/resources/defining-ipe/

3 CDS. Rapport du groupe de travail «Nouveaux modèles de soins pour la médecine de premier recours». 2012. www.gdk-cds.ch/fileadmin/docs/public/gdk/Aktuelles/ MMtlg/BT_Versmod_pub_20120402_f.pdf

4 D'Amour D, Oandasan I. Interprofessionality as the field of interprofessional practice and interprofessional education: An emerging concept. J Interprof Care. 2005;19.Suppl 1:8-20.

5 ASSM. Charte «Collaboration entre les professions de la santé». Novembre 2014. 